\title{
The activation of p38MAPK and JNK pathways in bovine herpesvirus 1 infected MDBK cells
}

\author{
Liqian Zhu ${ }^{1 \dagger}$, Chen Yuan ${ }^{1 \dagger}$, Liyuan Huang ${ }^{1}$, Xiuyan Ding ${ }^{1,2}$, Jianye Wang ${ }^{1}$, Dong Zhang ${ }^{1}$ and Guoqiang Zhu ${ }^{1 *}$
}

\begin{abstract}
We have shown previously that BHV-1 infection activates Erk1/2 signaling. Here, we show that BHV-1 provoked an early-stage transient and late-stage sustained activation of JNK, P38MAPK and c-Jun signaling in MDBK cells. C-Jun phosphorylation was dependent on JNK. These early events were partially due to the viral entry process. Unexpectedly, reactive oxygen species were not involved in the later activation phase. Interestingly, only activated JNK facilitated the viral multiplication identified through both chemical inhibitor and siRNA. Collectively, this study provides insight into our understanding of early stages of BHV-1 infection.
\end{abstract}

\section{Introduction}

Bovine herpesvirus 1 (BHV-1), an enveloped virus belonging to the alphaherpesvirus subfamily, infects cattle of all ages and breeds worldwide. BHV-1-induced immune suppression initiates the secondary bacterial infection and leads to bovine respiratory disease complex, ultimately resulting in high mortality $[1,2]$. The viral infection may also result in abortions, inflammation, conjunctivitis, and severe neonatal diseases. It costs the US cattle industry approximately 3 billion dollars annually [3].

Mitogen-activated protein kinases (MAPK), a family of serine/threonine protein kinases, are mainly divided into three family members including the extracellular signalregulated kinase 1 and 2 (Erk1/2), c-Jun NH2-terminal kinase (JNK) and p38MAPK $[4,5]$. They phosphorylate specific substrates at serine and/or threonine residues, and thereby transduce signals from the cell membrane to the nucleus in response to a wide range of stimuli, to

\footnotetext{
*Correspondence: yzgqzhu@yzu.edu.cn

${ }^{\dagger}$ Liqian Zhu and Chen Yuan are co-first authors

${ }^{1}$ College of Veterinary Medicine and Jiangsu Co-innovation

Center for Prevention and Control of Important Animal Infectious

Diseases and Zoonoses, Yangzhou University, 48 Wenhui East Road,

Yangzhou 225009, China

Full list of author information is available at the end of the article
}

participate in a diverse array of cellular programs including cell mitosis, proliferation, motility, metabolism, and other fundamental biological processes [6, 7]. Accumulated evidence indicates that MAPK pathways are involved in inflammatory response via activating the target genes of inflammatory mediators [8-10]. Moreover, inhibitors targeting $\mathrm{p} 38 \mathrm{MAPK}$ and JNK pathways have been developed for anti-inflammatory therapeutics, and the data from preclinical treatments have validated their prominent anti-inflammatory effect [11].

Since the MAPK cascades broadly regulate cellular biology function, it is not surprising that they are involved in the pathological responses of hosts to viral infection. For example, MAPK pathways were implicated in inflammatory response by the infection of influenza virus and HSV-1 [12-15]. The employment of MAPK inhibitors emerges as an attractive strategy to reduce both viral load and the level of pro-inflammatory cytokines to definitely control viral infection. We know that BHV-1 infection activates MAPK/Erk1/2 signaling in MDBK cells [16]. However, little is known about the response of p38MAPK and JNK in BHV-1 infection.

The aim of this study was to determine whether BHV-1 infection could alter p38MAPK and JNK pathways in MDBK cells. We found that BHV-1 infection of MDBK cells indeed activated both $\mathrm{p} 38 \mathrm{MAPK}$ and JNK pathways. 
However, only the JNK pathway was essential to viral replication. We also defined that c-Jun was exclusively activated by viral infection through JNK. Unexpectedly, BHV-1 infection-activated MAPK pathways was not through a reactive oxygen species (ROS)-dependent mechanism, though ROS is widely reported to be an activator of MAPK pathways during numerous virus infections, such as by HSV-1 $[17,18]$. These studies partially address the importance of MAPK pathways in BHV-1 infection induced inflammatory response.

\section{Materials and methods \\ Antibodies and reagents}

Antibodies against phospho-JNK (Thr183/Tyr185), phospho-p38MAPK (Thr180/Tyr182), Phospho-p44/42 MAPK (Erk1/2) (Thr202/Tyr204), phospho-c-Jun (Ser73), JNK, p38MAPK, p44/42 MAPK (Erk1/2), c-Jun, and GAPDH, as well as HRP labeled secondary antibodies anti-mouse IgG or anti-rabbit IgG were purchased from Cell Signaling Technology (Beverly, MA, USA). $N$-Acetyl-L-cysteine (NAC) was bought from SigmaAldrich (St. Louis, MO, USA). U0126, SB203580, and SP600125 were purchased from Cell Signaling Technology. BHV-1 VP16 antibody is kindly provided by Prof. Vikram Misra at the University of Saskatchewan [19].

\section{Virus and cell cultures}

MDBK cells (provided by Dr Leonard J. Bello, University of Pennsylvania) were maintained at $37{ }^{\circ} \mathrm{C}$ in $5 \% \mathrm{CO}_{2}$ in DMEM (Gibco BRL) supplemented with $10 \%$ horse serum (HyClone Laboratories, Logan, UT, USA). BHV-1 Colorado1 strain (provided by Dr Leonard J. Bello, University of Pennsylvania) used for this study was propagated in MDBK cells. Aliquots of virus stocks were stored at $-70{ }^{\circ} \mathrm{C}$ until use. The virus was titrated in MDBK cells with results expressed as $\mathrm{TCID}_{50}$ calculated using the Reed-Muench formula. The inactivation of BHV-1 by UV-irradiation was performed as previously described [16]. Effective inactivation of viruses was confirmed by virus titer assay in MDBK cells.

\section{siRNA knockdown}

siRNA1-3 targeting JNK1, and siRNA4-6 targeting JNK2 as well as the control siRNA were purchased from Genepharma (Shanghai, China). SiRNA transfection was performed with transfection reagent siRNA-Mate (Genepharma) according to the manufacturer's specifications. Efficiency of these siRNA were characterized by Western blotting.

\section{Inhibition of viral replication by chemical inhibitors}

Confluent MDBK cells in 24-well plates were pretreated with the detected inhibitors at the indicated concentrations for $1 \mathrm{~h}$ at $37^{\circ} \mathrm{C}$, followed by BHV-1 infection at MOI of 1 for $1 \mathrm{~h}$. After extensive washing with PBS, the cells were replaced with fresh medium DMEM $(400 \mu \mathrm{L})$ with or without chemicals and cultured in a $\mathrm{CO}_{2}$ incubator at $37^{\circ} \mathrm{C}$. The virus yield was titrated by $\mathrm{TCID}_{50}$ assay.

\section{Western blot analysis}

Monolayers of MDBK cells in 60-mm dishes were serum starved overnight, mock-infected or infected with BHV-1 $(\mathrm{MOI}=10)$ at $37{ }^{\circ} \mathrm{C}$ for $0.5,1,2,4,8,12$ and $24 \mathrm{~h}$. Cell lysates were prepared with lysis buffer (1\% Triton X-100, $50 \mathrm{mM}$ sodium chloride, $1 \mathrm{mM}$ EDTA, $1 \mathrm{mM}$ EGTA, $20 \mathrm{mM}$ sodium fluoride, $20 \mathrm{mM}$ sodium pyrophosphate, $1 \mathrm{mM}$ phenylmethylsulfonyl fluoride, $0.5 \mathrm{~g} / \mathrm{mL}$ leupeptin, $1 \mathrm{mM}$ benzamidine, and $1 \mathrm{mM}$ sodium orthovanadate in $20 \mathrm{mM}$ Tris- $\mathrm{HCl}, \mathrm{pH} 8.0$ ) at the indicated time points. To analyze the effect of detected inhibitors on the designated signaling, MDBK cells were exposed to the corresponding chemicals through virus infection plus a pretreatment for $1 \mathrm{~h}$ before virus inoculation. To analyze the effect of siRNA on the designated signaling, MDBK cells were transfected with siRNA and incubated for $48 \mathrm{~h}$, then infected with BHV-1 for the indicated time length at $37^{\circ} \mathrm{C}$.

Cell lysates were separated on 8 or 10\% SDS-polyacrylamide gels and transferred to a polyvinylidene difluoride (PVDF) membrane (Bio-rad, CA, USA). After blocking with $5 \%$ nonfat milk in Tris-buffered saline (TBS) buffer containing $0.05 \%$ Tween 20 (TBST), the membrane was incubated with respective primary antibodies, and followed by HRP-conjugated secondary antibodies in the blocking reagent. After extensive washing with TBST, immune reactive bands were detected by film exposure after enhanced chemiluminescence (ECL) reaction (Millipore, USA).

\section{Results}

We previously reported that BHV-1 infection induces the activation of MAPK/Erk $1 / 2$ signaling in MDBK cells [16]. To identify whether JNK and p38MAPK signaling were involved in BHV-1 infection of MDBK cells, we first examined the kinetics of phosphorylated JNK and $\mathrm{p} 38 \mathrm{MAPK}$ after BHV-1 virus infection using Western blotting. To see the burst effect of virus infection on the detected signaling pathways, serum starved MDBK cells were infected with BHV-1 at a high MOI of 10 . As a result, the levels of both phospho-JNK (Thr183/ Tyr185) and phospho-p38MAPK (Thr180/Tyr182) were dramatically elevated in a two-tiered manner following BHV-1 infection (Figure 1A). The first tier was transiently increased at the early stage of virus infection (Figure $1 \mathrm{~A}, 0.5 \mathrm{hpi})$. The second wave was sustained at the 


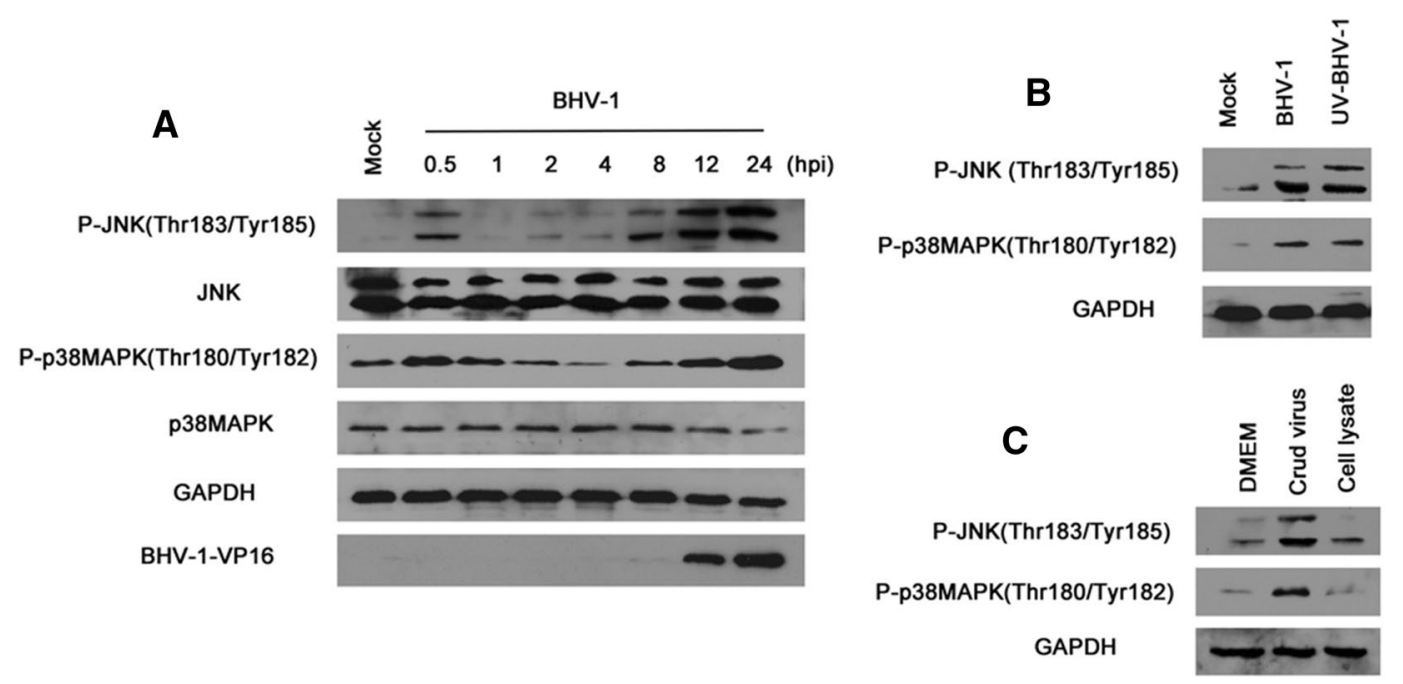

Figure 1 Biphasic activation of both JNK and p38MAPK pathway in BHV-1 infected MDBK cells. A Time course of altered phosphoJNK(Thr183/Tyr185) and phosphor-p38MAPK(Thr180/Tyr182) following BHV-1 infection. Growth arrested MDBK cells were mock infected or infected with BHV-1 at an MOI of 10. At the indicated time points, cells were lysed and subjected to Western blotting analysis using the indicated antibodies. B UV-irradiation inactivated virus also induced activation of both JNK and p38MAPK. MDBK cells were infected with BHV-1 (MOI $=10)$ or with equal amounts of UV-irradiation inactivated virus for $30 \mathrm{~min}$. The cells were lysed for Western blotting. C Uninfected cell lysates could not stimulate p38MAPK and JNK signaling. To get uninfected cell lysates and crude virus, MDBK cells were mock infected or infected with BHV-1 (MOI = 1) without serum in the medium. At $48 \mathrm{hpi}$, the cell cultures were subjected to frozen-thawing and centrifugation at $15000 \mathrm{rpm}$ for $10 \mathrm{~min}$. The supernatants were collected and used for the treatment of MDBK cells. At $0.5 \mathrm{~h}$ post-treatment, the cells were lysed for Western blotting analysis. Data are representative results of three independent experiments.

later stage of infection (Figure 1A, 12 and $24 \mathrm{hpi}$ ). Obviously, the alteration of phosphorylated p38MAPK or JNK was neither due to overexpression of total p38MAPK (or JNK) nor to a high amount of protein loading (Figure 1A). Here, the lytic cycle viral protein VP16 of BHV-1 was detected to identify the kinetics of viral infection. Interestingly, the kinetics of the second tier of activated p38MAPK and JNK correlated with that of viral protein VP16 expression (Figure 1A). It is highly possible that the de novo viral protein expression and/or DNA replication account for the second tier of activated p38MAPK and JNK.

Considering that the early-transient activation of both p38MAPK and JNK appeared as early as $0.5 \mathrm{hpi}$, UVirradiation inactivated virus could enter cells but not initiate subsequent genomic replication nor translation. We employed the UV-irradiation inactivated virus to explore the role of the viral entry process on these activations. UV-irradiation inactivated viruses were generated with infectious virus exposed to a $30 \mathrm{~W}$ UV light at a distance of $10 \mathrm{~cm}$ for $1 \mathrm{~h}$. Complete inactivation of virus was confirmed with a virus titer assay (data not shown). MDBK cells were inoculated with the infectious virus $(\mathrm{MOI}=10)$ or equal amounts of UV-inactivated virus in parallel for $0.5 \mathrm{~h}$. As shown in Figure 1B, UV-irradiated BHV-1 induced similar levels of p38MAPK and JNK activation when compared to functional BHV-1, suggesting that an early step(s) of viral infection would be responsible for these events.

Though we generated the virus stocks without serum in the cell culture, both cell debris or growth factors were still present in the crude virus, which may have stimulated JNK and p38MAPK signaling. The supernatant from uninfected cell culture was obtained in parallel to the virus stock. MDBK cells were treated with either supernatant of uninfected cell culture or crude virus at $37{ }^{\circ} \mathrm{C}$ for $0.5 \mathrm{~h}$, the phosphorylation of both JNK and p38MAPK was detected using Western blotting. As a result, the supernatant of uninfected cell culture could not induce apparent activation of both JNK and p38MAPK (Figure 1C). It ruled out the possibility that the activation of JNK and p38MAPK signaling by UV-inactivated virus was due to the cellular debris or the presence of growth factors in the medium.

To investigate the role of p38MAPK and JNK pathways in viral amplification, chemical inhibitors SP600125 and SB203580 specific for JNK and p38MAPK, were respectively employed to treat MDBK cells during the virus infection. Proper concentration for each inhibitor that showed no cytotoxicity to MDBK cells was selected based on the specifications and 3-(4,5-dimethyl-2-thiazolyl)-2,5-diphenyl-2H-tetrazolium bromide 
(MTT) assay (data not shown). The treatment with JNK specific inhibitor SP600125 at 6.25, 12.5 and $25 \mu \mathrm{M}$ strongly reduced the virus production ranging from $\sim 1$ to $\sim 1.6$ logs (Figure 2A). Although p38MAPK was activated by BHV-1 infection, the p38MAPK specific inhibitor SB203580 showed no apparent effect on viral replication (Figure 2B). The data suggest that JNK pathways play an important role in viral amplification.

The JNK are encoded by three separate genes (JNK1, 2 , and 3), which are spliced alternatively to create 10 JNK isoforms that are either 54 or $46 \mathrm{kDa}$ in size [20]. In mammalians, JNK1 and JNK2 are widely expressed, whereas JNK3 expression is largely restricted to the brain $[21,22]$. Chemical inhibitors might exert off-target effects. We used siRNA-mediated knock down to address the specific role of JNK in BHV-1 replication. MDBK cells were transiently transfected with siRNA1-3 targeting JNK1, and siRNA4-6 targeting JNK2 as well as the control siRNA (provided by Genepharma, Shanghai, China). Of these detected siRNA, only siRNA1 could apparently reduce the expression of JNK as determined by Western blotting (Figure 2C). Next, MDBK cells in 24-well plates were transfected with siRNA1, siRNA2 and control siRNA, respectively, and at $48 \mathrm{~h}$ post-transfection they were infected with BHV-1 (MOI = 1). The virus yield was determined at $24 \mathrm{hpi}$ with $\mathrm{TCID}_{50}$ assay. As a result, comparing to either control siRNA or siRNA2, siRNA1 moderately decreased viral titer by $\sim 0.5 \log$ (Figure 2D). Although the inhibitory effect of siRNA1 was not as strong as the inhibitor SP600125 on viral replication, knockdown of JNK1 consistently decreased the virus infection. In addition, siRNA transfection reduced the level of phosphorylated JNK (Figure 2E), which corroborated the result that BHV-1 infection activated JNK, and knockdown of JNK1 reduced BHV-1 replication. Collectively, these results imply that JNK1 may be involved in BHV-1 replication.

Upon activation, the phosphorylated JNK form dimers and enter the nucleus to activate target genes through its effects on c-Jun, ATF-2, and other transcription factors [23]. We therefore subsequently monitored the kinetics of activated c-Jun during BHV-1 infection through a Western blotting assay. The elevated levels of phosphorylated c-Jun (Ser73) were observed at both early and later stages of virus infection (Figure 3A). Exposure to UV-inactivated virus rendered an early event of c-Jun phosphorylation, as determined at $0.5 \mathrm{hpi}$ at a level comparable to that obtained with functional virus (Figure 3B). It implies that BHV-1 infection activated c-Jun pathways, and viral early entry steps could be responsible for the early wave of activation.

Although c-Jun indeed is a canonical downstream target of JNK, accumulated data has shown that c-Jun can also be activated by other MAPK signaling axes such as by p38MAPK and Erk1/2. For example, JNK independent phosphorylation of c-Jun has been demonstrated in numerous cells stimulated with phorbol 12-myristate 13-acetate (PMA) [24], and c-Jun-dependent microglial inflammatory response following irradiation is mediated by Erk1/2 but not by JNK [25]. Here, we chemically inhibited JNK signaling with SP600125, p38MAPK signaling with SB203580 and Erk1/2 signaling with U0126, to elucidate whether there was a dialog between c-Jun and p38MAPK and Erk1/2. MDBK cells were pretreated with compounds at indicated concentrations for $1 \mathrm{~h}$ and then infected with BHV-1 $(\mathrm{MOI}=10)$ in the presence of inhibitors. At 0.5 and $24 \mathrm{hpi}$, the cells were lysed and p-c-Jun was detected by Western blotting. Interestingly, BHV-1 induced phosphorylation of c-Jun was uniquely inhibited by JNK inhibitor SP600125 (Figures 3B and C). This indicates that JNK is the unique stimulator for BHV-1-activated c-Jun signaling. Similarly, it has been reported that treatment of HSV-1-infected Vero cells with SB203580 results in minor effects on c-Jun activation [26].

Reactive oxygen species are key signaling molecules that play an important role in the progression of inflammatory disorders [27]. Our recent data indicate that BHV-1 infection stimulates ROS production to facilitate viral infection and mediate mitochondrial dysfunction [28]. HSV-1 is genetically close to BHV-1, which stimulates MAPK signaling with a ROS-dependent manner [10]. Therefore, the relation between ROS production and MAPK activation induced by BHV-1 infection was investigated. MDBK cells were treated with ROS scavenger NAC [29] at a concentration of 5 and $1 \mathrm{mM}$, respectively, and infected with virus $(\mathrm{MOI}=10)$. At $24 \mathrm{hpi}$ the cells were lysed for Western blotting to detect the phosphorylation of p38MAPK, JNK, Erk1/2 and c-Jun. As a result, none of them were blocked by ROS scavenger NAC (Figure 4). This suggests that BHV-1 stimulated the three MAPK signaling, p38MAPK, Erk1/2 and JNK/cJun with a ROS-independent manner, which was distinct from that of the genetic closely related HSV-1.

\section{Discussion}

The canonical MAPK pathways implicated in various inflammatory responses have been reported to be modified by diverse viruses to support productive replication, control cell proliferation, suppress cell apoptosis, or induce cytokine production and inflammation [10, $30,31]$. For example, the activation of p38MAPK and Erk $1 / 2$ is required for the nuclear export of viral ribonucleoprotein complexes [32, 33]. Here, we established that both p38MAPK and JNK signaling were activated in BHV-1-infected MDBK cells (Figure 1). Together with 
A

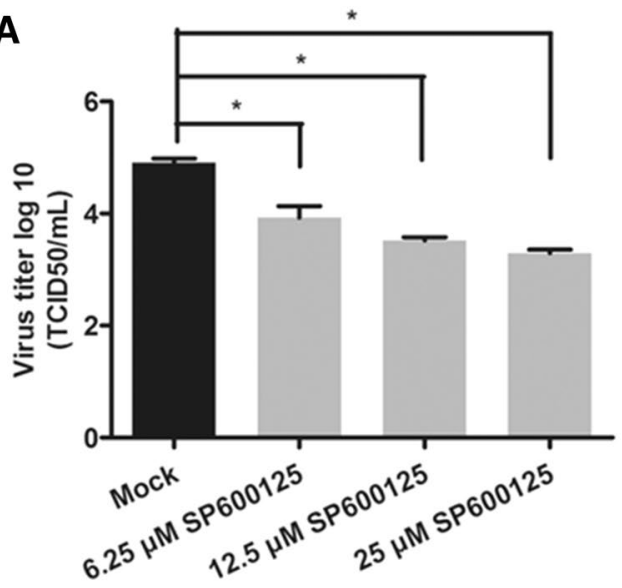

C

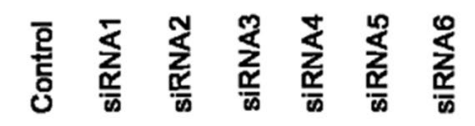

JNK

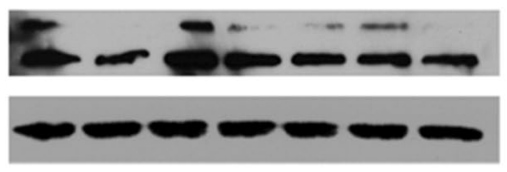

GAPDH

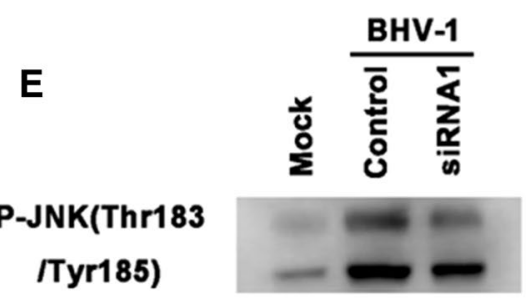

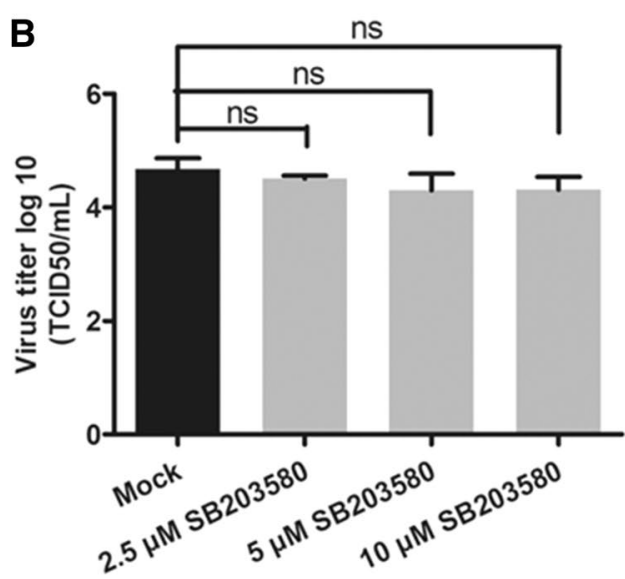

ns

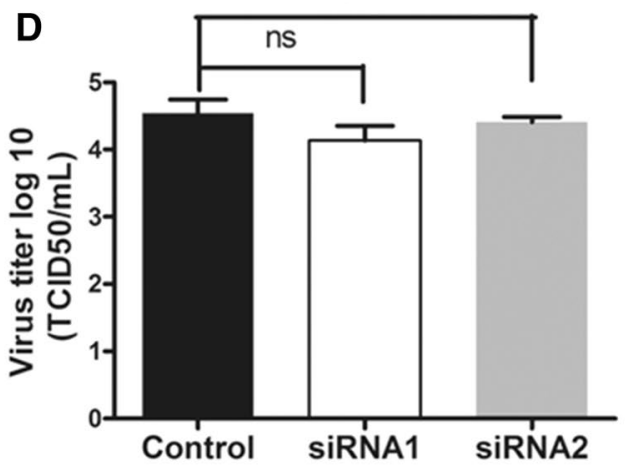

B-Actin

Figure 2 The JNK but not p38MAPK signaling related to BHV-1 replication. A B JNK inhibitor inhibited BHV-1 replication. MDBK cells were first pretreated with inhibitor SP600125 A specific for JNK or inhibitor SB203580 B specific for p38MAPK at various concentrations for $1 \mathrm{~h}$, and then infected with BHV-1 at an MOI of 1. At 24 hpi, virus yield was titrated using the TCID 50 assay. C Screening siRNA target for JNK1 and JNK2. MDBK cells in 6-well plates were transfected with the indicated siRNA of $100 \mathrm{pM}$ using transfection reagent siRNA-Mate (Genepharma) according to the specifications. At $48 \mathrm{~h}$ post transfection, the cells were lysed and subjected to Western blotting with antibody against JNK. D Knock down of the expression of JNK1 with siRNA1 moderately inhibited BHV-1 replication. MDBK cells in 24-well plates were transfected with siRNA1, siRNA3 and control siRNA of $20 \mathrm{pM}$ using transfection reagent siRNA-Mate. At $48 \mathrm{~h}$ post transfection, the cells were infected with BHV-1 at MOl of 1 for $1 \mathrm{~h}$, after extensive washing with PBS, fresh medium was replaced for further incubation of $24 \mathrm{~h}$. Virus yield was tittered with $\mathrm{TCID}_{50}$. The data are from three independent experiments. Statistical analyses were performed using the Student's t test ( $P<0.05$ vs. control). E Knockdown JNK1 by siRNA1 reduced JNK1 phosphorylation induced by BHV-1 infection. MDBK cells in 6-well plates were transfected with siRNA1 and control siRNA, respectively. At $48 \mathrm{~h}$ post-transfection, the cells were infected with BHV-1 at MOI of 10 for $0.5 \mathrm{~h}$. The cell lysates were prepared for Western blotting analysis. Data are representative of two repeats.

our previous data [16], we knew that in BHV-1 infected MDBK cells the three canonical MAPK signaling including p38 MAPK, JNK and Erk1/2 were activated. BHV-1 and HSV-1 belong to the Alphaherpesvirinae subfamily and share a number of biological properties. However,
HSV-1 infection activates both p38 MAPK and JNK signaling, but reduces Erk1/2 signaling [14, 34, 35]. Obviously, these MAPK pathways were differentially manipulated by BHV-1 and HSV-1. It is reasonable that the discriminatory controlling of MAPK pathways would 


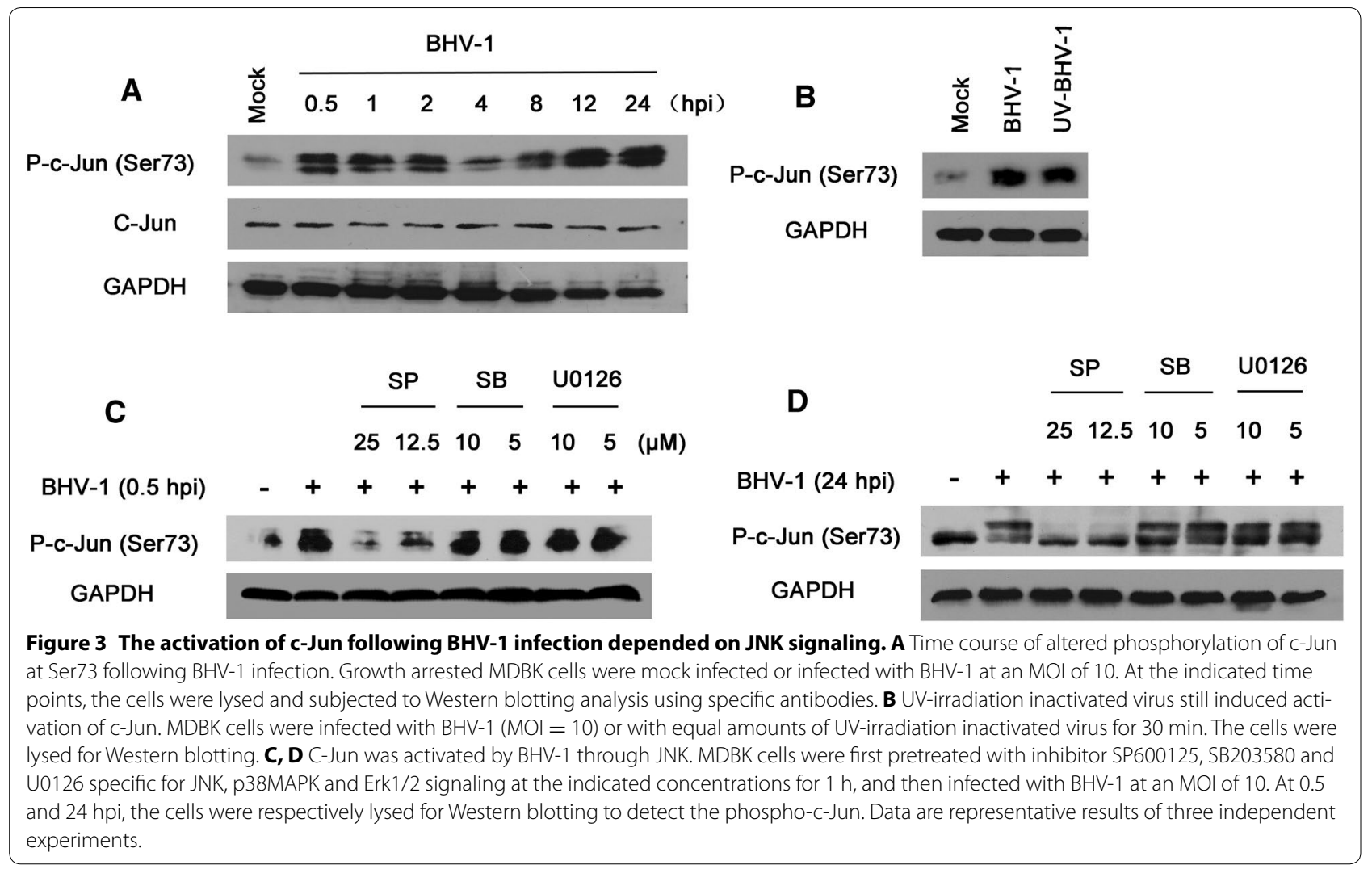

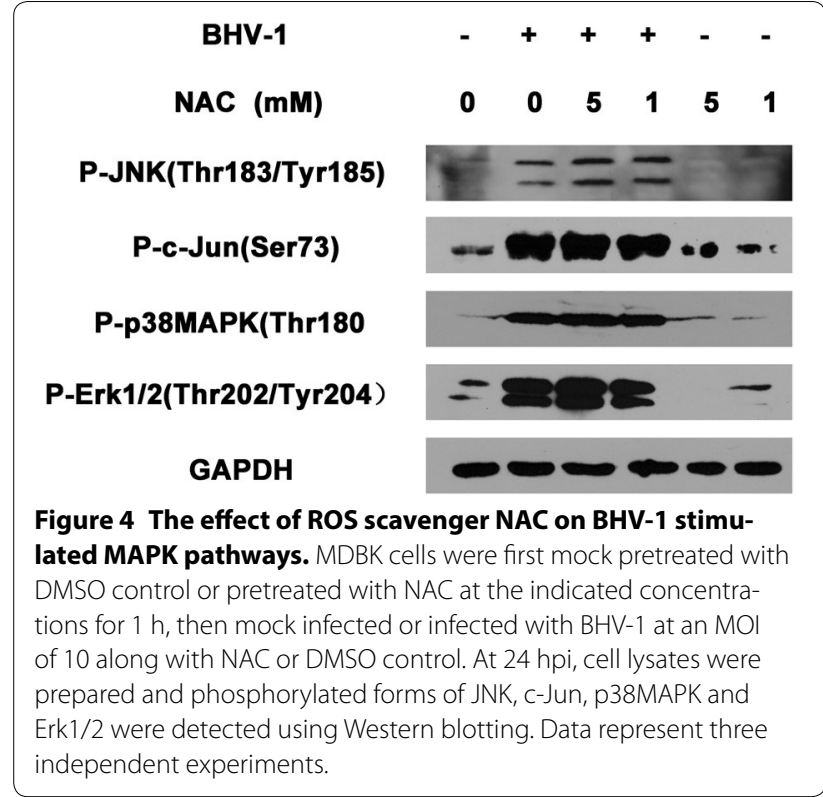

produce different effects on virus pathogenicity. In the future it would be interesting to study the mechanisms of the differential manipulation of MAPK pathways by these two viruses.
Since the UV-inactivated viral particles could enter host cells but not complete subsequent gene transcription, they could still activate these MAPK signaling at 0.5 hpi. Based on this data and our previous report [16], we suggest that the viral entry process may partially account for the enhanced phosphorylation of these three MAPK signaling. ROS are important inflammatory mediators, which are recognized as secondary messengers to activate a variety of cellular signaling pathways such as p38MAPK and Erk1/2 after HSV-1 infection of murine microglial cells [10]. We recently reported that BHV-1 infection increases ROS production, which contributes to viral replication [28]. Considering that BHV-1 and HSV-1 are genetically closely related, ROS is a putative component responsible for BHV-1 activated MAPK pathways. However, here we found that ROS was not accounted for BHV-1-stimulated phosphorylation of p38MAPK, Erk1/2 and JNK (Figure 4). So the activation of these MAPK pathways by BHV-1was not mediated by ROS.

JNK activation may exert viral-supportive or antiviral effect for diverse viruses. For example, JNK knockout mouse embryonic fibroblasts (MEF) were more susceptible to oncolytic vaccinia virus infection than wild-type MEF [36]. In contrast, JNK inhibitor SP600125 possesses a strong inhibitory effect on viral replication of either 
highly pathogenic avian virus strain A/FPV/Bratislava/79 (H7N7) or the pandemic swine-origin influenza virus A/ Hamburg/4/09 (H1N1v) [37]. Here, we elucidated that only JNK was required to support BHV-1 replication, but p38MAPK could not (Figures 2A, B, D). Interestingly, both P38 MAPK and JNK pathways are important for HSV-1 gene expression and viral propagation $[26,34,38]$. So the MAPK pathways are discriminately controlled by BHV-1 and HSV-1 for viral replication.

In summary, for the first time we elucidated that in BHV-1 infected MDBK cells, all three major MAPK pathways are activated in response to viral infection, but JNK signaling was uniquely required for viral replication. Interestingly, we provide evidence that BHV-1 activated the MAPK pathways with a ROS-independent mechanism, which was different from that with HSV-1. The potential participation of these pathways in diverse processes of BHV-1 infection would provide valuable information towards comprehending the infection and inflammatory mechanism of BHV-1 infection in bovines.

\section{Competing interests}

The authors declare that they have no competing interests.

\section{Authors' contributions}

LZ participated in design of the study, analyzed the data and prepared the manuscript. CY, LH and XD carried out the experiments, DZ cultured the cells. JW revised the manuscript, GZ coordinated the research. All authors read and approved the final manuscript.

\section{Acknowledgements}

The authors are grateful to Dr Leonard J. Bello, University of Pennsylvania, for providing the MDBK cells and the Colorado 1 strain of BHV-1, and thanks for Prof. Vikram Misra from University of Saskatchewan for kindly providing the antibody against BHV-1 VP16. This research was supported by Chinese National Science Foundation Grant (No. 31472172), the Priority Academic Program Development of Jiangsu Higher Education Institutions (PAPD and TAPP). Partly supported by the Grant Nos. BE2014358 and 14KJA230001 from Jiangsu province.

\section{Author details}

${ }^{1}$ College of Veterinary Medicine and Jiangsu Co-innovation Center for Prevention and Control of Important Animal Infectious Diseases and Zoonoses, Yangzhou University, 48 Wenhui East Road, Yangzhou 225009, China. ${ }^{2}$ The Test Center of Yangzhou University, 48 Wenhui East Road, Yangzhou 225009, China.

Received: 24 March 2016 Accepted: 28 June 2016

Published online: 02 September 2016

\section{References}

1. Tikoo SK, Campos M, Babiuk LA (1995) Bovine herpesvirus 1 (BHV-1): biology, pathogenesis, and control. Adv Virus Res 45:191-223

2. Muylkens B, Thiry J, Kirten P, Schynts F, Thiry E (2007) Bovine herpesvirus 1 infection and infectious bovine rhinotracheitis. Vet Res 38:181-209

3. Jones C, Chowdhury S (2007) A review of the biology of bovine herpesvirus type $1(\mathrm{BHV}-1)$, its role as a cofactor in the bovine respiratory disease complex and development of improved vaccines. Anim Health Res Rev 8:187-205

4. Hazzalin CA, Mahadevan LC (2002) MAPK-regulated transcription: a continuously variable gene switch? Nat Rev Mol Cell Biol 3:30-40
5. Dong W, Wei X, Zhang F, Hao J, Huang F, Zhang C, Liang W (2014) A dual character of flavonoids in influenza a virus replication and spread through modulating cell-autonomous immunity by MAPK signaling pathways. Sci Rep 4:7237

6. Roux PP, Blenis J (2004) ERK and p38 MAPK-activated protein kinases: a family of protein kinases with diverse biological functions. Microbiol Mol Biol Rev 68:320-344

7. Wada T, Penninger JM (2004) Mitogen-activated protein kinases in apoptosis regulation. Oncogene 23:2838-2849

8. Panteva M, Korkaya H, Jameel S (2003) Hepatitis viruses and the MAPK pathway: is this a survival strategy? Virus Res 92:131-140

9. Xie J, Zhang S, Hu Y, Li D, Cui J, Xue J, Zhang G, Khachigian LM, Wong J, Sun L, Wang M (2014) Regulatory roles of c-jun in H5N1 influenza virus replication and host inflammation. Biochim Biophys Acta 1842:2479-2488

10. Hu S, Sheng WS, Schachtele SJ, Lokensgard JR (2011) Reactive oxygen species drive herpes simplex virus (HSV)-1-induced proinflammatory cytokine production by murine microglia. J Neuroinflamm 8:123

11. Kaminska B (2005) MAPK signalling pathways as molecular targets for anti-inflammatory therapy-from molecular mechanisms to therapeutic benefits. Biochim Biophys Acta 1754:253-262

12. Pleschka S, WolffT, Ehrhardt C, Hobom G, Planz O, Rapp UR, Ludwig S (2001) Influenza virus propagation is impaired by inhibition of the Raf/ MEK/ERK signalling cascade. Nat Cell Biol 3:301-305

13. Wang X, Tan J, Zoueva O, Zhao J, Ye Z, Hewlett I (2014) Novel pandemic influenza A (H1N1) virus infection modulates apoptotic pathways that impact its replication in A549 cells. Microbes Infect 16:178-186

14. Chuluunbaatar U, Roller R, Mohr I (2012) Suppression of extracellular signal-regulated kinase activity in herpes simplex virus 1-infected cells by the Us3 protein kinase. J Virol 86:7771-7776

15. Gaur P, Munjhal A, Lal SK (2011) Influenza virus and cell signaling pathways. Med Sci Monit 17:RA148-154

16. Zhu L, Ding X, Zhu X, Meng S, Wang J, Zhou H, Duan Q, Tao J, Schifferli DM, Zhu G (2011) Biphasic activation of PI3 K/Akt and MAPK/Erk1/2 signaling pathways in bovine herpesvirus type 1 infection of MDBK cells. Vet Res 42:57

17. Chen D, Su A, Fu Y, Wang X, Lv X, Xu W, Xu S, Wang H, Wu Z (2015) Harmine blocks herpes simplex virus infection through downregulating cellular NF-kappaB and MAPK pathways induced by oxidative stress. Antivir Res 123:27-38

18. Chen X, Qiao H, Liu T, Yang Z, Xu L, Xu Y, Ge HM, Tan RX, Li E (2012) Inhibition of herpes simplex virus infection by oligomeric stilbenoids through ROS generation. Antivir Res 95:30-36

19. Misra V, Bratanich AC, Carpenter D, O'Hare P (1994) Protein and DNA elements involved in transactivation of the promoter of the bovine herpesvirus (BHV) $1 \mathrm{IE}-1$ transcription unit by the BHV alpha gene trans-inducing factor. J Virol 68:4898-4909

20. Tsuiki H, Tnani M, Okamoto I, Kenyon LC, Emlet DR, Holgado-Madruga M, Lanham IS, Joynes CJ, Vo KT, Wong AJ (2003) Constitutively active forms of c-Jun $\mathrm{NH} 2$-terminal kinase are expressed in primary glial tumors. Cancer Res 63:250-255

21. Davis RJ (2000) Signal transduction by the JNK group of MAP kinases. Cell 103:239-252

22. Bogoyevitch MA (2006) The isoform-specific functions of the c-Jun $\mathrm{N}$-terminal kinases (JNKs): differences revealed by gene targeting. BioEssays 28:923-934

23. Leppa S, Bohmann D (1999) Diverse functions of JNK signaling and c-Jun in stress response and apoptosis. Oncogene 18:6158-6162

24. Humar M, Loop T, Schmidt R, Hoetzel A, Roesslein M, Andriopoulos N, Pahl HL, Geiger KK, Pannen BH (2007) The mitogen-activated protein kinase p38 regulates activator protein 1 by direct phosphorylation of c-Jun. Int J Biochem Cell Biol 39:2278-2288

25. Deng Z, Sui G, Rosa PM, Zhao W (2012) Radiation-induced c-Jun activation depends on MEK1-ERK1/2 signaling pathway in microglial cells. PLoS One 7:e36739

26. Karaca G, Hargett D, McLean TI, Aguilar JS, Ghazal P, Wagner EK, Bachenheimer SL (2004) Inhibition of the stress-activated kinase, p38, does not affect the virus transcriptional program of herpes simplex virus type 1. Virology 329:142-156

27. Mittal M, Siddiqui MR, Tran K, Reddy SP, Malik AB (2014) Reactive oxygen species in inflammation and tissue injury. Antioxid Redox Signal 20:1126-1167 
28. Zhu L, Yuan C, Zhang D, Ma Y, Ding X, Zhu G (2016) BHV-1 induced oxidative stress contributes to mitochondrial dysfunction in MDBK cells. Vet Res 47:47

29. Kang S, Lu K, Leelawattanachai J, Hu X, Park S, Park T, Min IM, Jin MM (2013) Virus-mimetic polyplex particles for systemic and inflammation-specific targeted delivery of large genetic contents. Gene Ther 20:1042-1052

30. Mizumura K, Hashimoto S, Maruoka S, Gon Y, Kitamura N, Matsumoto K, Hayashi S, Shimizu K, Horie T (2003) Role of mitogen-activated protein kinases in influenza virus induction of prostaglandin E2 from arachidonic acid in bronchial epithelial cells. Clin Exp Allergy 33:1244-1251

31. Pleschka S (2008) RNA viruses and the mitogenic Raf/MEK/ERK signal transduction cascade. Biol Chem 389:1273-1282

32. Nencioni L, De Chiara G, Sgarbanti R, Amatore D, Aquilano K, Marcocci ME, Serafino A, Torcia M, Cozzolino F, Ciriolo MR, Garaci E, Palamara AT (2009) Bcl-2 expression and p38MAPK activity in cells infected with influenza A virus: impact on virally induced apoptosis and viral replication. J Biol Chem 284:16004-16015

33. Marjuki H, Alam MI, Ehrhardt C, Wagner R, Planz O, Klenk HD, Ludwig S. Pleschka S (2006) Membrane accumulation of influenza A virus hemagglutinin triggers nuclear export of the viral genome via protein kinase Calpha-mediated activation of ERK signaling. J Biol Chem 281:16707-16715

34. McLean TI, Bachenheimer SL (1999) Activation of CJUN N-terminal kinase by herpes simplex virus type 1 enhances viral replication. J Virol 73:8415-8426

35. Hargett D, McLean T, Bachenheimer SL (2005) Herpes simplex virus ICP27 activation of stress kinases JNK and p38. J Virol 79:8348-8360

36. Hu W, Hofstetter W, Guo W, Li H, Pataer A, Peng HH, Guo ZS, Bartlett DL, Lin A, Swisher SG, Fang B (2008) JNK-deficiency enhanced oncolytic vaccinia virus replication and blocked activation of double-stranded RNA-dependent protein kinase. Cancer Gene Ther 15:616-624

37. Nacken W, Ehrhardt C, Ludwig S (2012) Small molecule inhibitors of the c-Jun N-terminal kinase (JNK) possess antiviral activity against highly pathogenic avian and human pandemic influenza A viruses. Biol Chem 393:525-534

38. Zachos G, Clements B, Conner J (1999) Herpes simplex virus type 1 infection stimulates $\mathrm{p} 38 / \mathrm{c}$-Jun $\mathrm{N}$-terminal mitogen-activated protein kinase pathways and activates transcription factor AP-1. J Biol Chem 274:5097-5103

\section{Submit your next manuscript to BioMed Central and we will help you at every step:}

- We accept pre-submission inquiries

- Our selector tool helps you to find the most relevant journal

- We provide round the clock customer support

- Convenient online submission

- Thorough peer review

- Inclusion in PubMed and all major indexing services

- Maximum visibility for your research

Submit your manuscript at www.biomedcentral.com/submit 